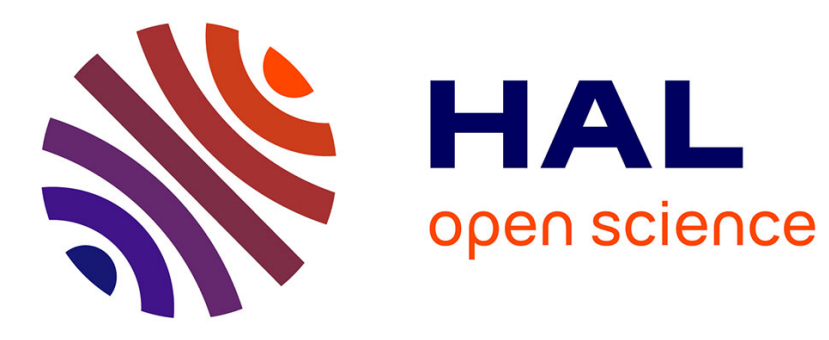

\title{
Securing Industrial Operators with Collaborative Robots: Simulation and Experimental Validation for a Carpentry task
}

\author{
Nassim Benhabib, Vincent Padois, David Daney
}

\section{- To cite this version:}

Nassim Benhabib, Vincent Padois, David Daney. Securing Industrial Operators with Collaborative Robots: Simulation and Experimental Validation for a Carpentry task. ICRA 2020 - IEEE International Conference on Robotics and Automation, May 2020, Paris, France. 10.1109/ICRA40945.2020.9197161 . hal-02418739v2

\section{HAL Id: hal-02418739 \\ https://hal.inria.fr/hal-02418739v2}

Submitted on 29 May 2020

HAL is a multi-disciplinary open access archive for the deposit and dissemination of scientific research documents, whether they are published or not. The documents may come from teaching and research institutions in France or abroad, or from public or private research centers.
L'archive ouverte pluridisciplinaire HAL, est destinée au dépôt et à la diffusion de documents scientifiques de niveau recherche, publiés ou non, émanant des établissements d'enseignement et de recherche français ou étrangers, des laboratoires publics ou privés. 


\title{
Securing Industrial Operators with Collaborative Robots: Simulation and Experimental Validation for a Carpentry task
}

\author{
Nassim Benhabib ${ }^{1}$ and Vincent Padois ${ }^{1}$ and David Daney ${ }^{1}$
}

\begin{abstract}
In this work, a robotic assistance strategy is developed to improve the safety in an artisanal task that involves a strong interaction between a machine-tool and an operator. Wood milling is chosen as a pilot task due to its importance in carpentry and its accidentogenic aspect. A physical model of the tooling process including a human is proposed and a simulator is thereafter developed to better understand situations that are dangerous for the craftsman. This simulator is validated with experiments on three subjects using an harmless mockup. This validation shows the pertinence of the proposed control approach for the collaborative robot used to increase the safety of the task.
\end{abstract}

\section{INTRODUCTION}

A wood shaper is a machining tool used to profile wooden parts (see fig. 1). During the milling process, a craftsman is handling a non homogeneous anisotropic material, in strong interaction with the cutting tool. It can be difficult to guarantee the stability of the part throughout the task. This can lead to tragic accidents ranging from bruises to severe cuts of the carpenter's limbs. According to a study conducted by J. Hester et al. [1], among 1200 apprentice carpenters interviewed, $80 \%$ of them have already observed a work accident. Similarly, in 2016, the French Institute for Craftsmen's Health and Safety (IRIS-ST) counted 5118 accidents for 61764 carpenters [2]. $27 \%$ of them were related to tooling tasks.

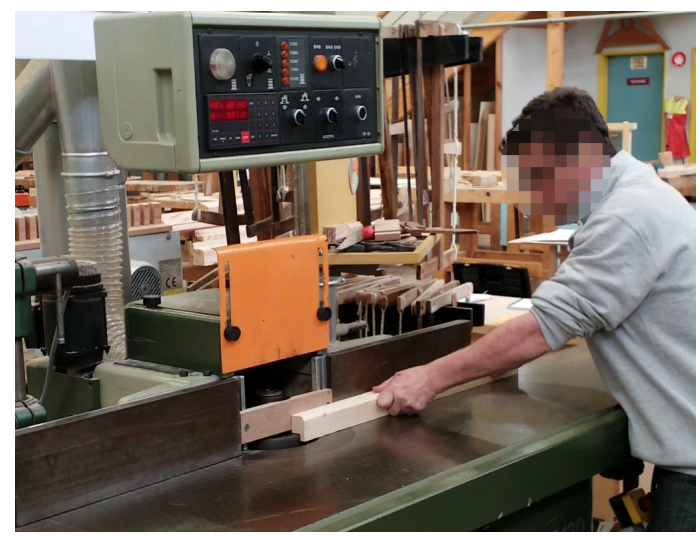

Fig. 1: Illustration of a wood milling task by a trained expert on a wood shaper. The left arm maintains lateral stability while the right arm (hidden on this picture) pushes the part in direction of the cutting tool.

Automated solutions for medium and large series production in the woodworking industry are not adapted to craft trades that require flexibility and adaptability. A conventional robotic approach is too expensive for many small businesses and removes the human

\footnotetext{
${ }^{1}$ Inria, Centre Bordeaux Sud-Ouest, Equipe AUCTUS Inria / IMS(Univ. Bordeaux, CNRS UMR5218), F-33405 Talence, France firstname. namedinria. fr.
}

from the loop. However, collaborative robotics potentially offers new possibilities for assisting carpenters, preserving their knowhow and sensations while making the task safer.

Several types of robotic assistance are generally envisioned [3]. One of the most commonly considered ones is comanipulation by gravity compensation and/or force amplification. For example, the authors of [4] propose that an upper limbs exoskeleton participates in reducing the strenuousness of some industrial tasks, the advantage of an exoskeleton being to act both on the posture while amplifying the user strength. This type of assistance is also considered in the construction industry for handling heavy loads [5]. In industrial domains where the workers are technical experts of complex manual tasks (i.e craftsmen), force amplification is also considered as a approach of interest. For example, Lamy et al. [6] have developed a robotic assistance solution for butcher cutting large pieces of meat in slaughter houses. While the impact on the worker is positive in terms of reduction of musculoskeletal disorders, the robot is not directly envisioned as way to protect the worker from potential cuts with the knife.

While these research works provide examples of how collaborative robots can provide physical assistance to workers, this assistance is in general not designed with immediate safety in mind. In fact, prototyping robotic solutions dedicated to immediate safety in highly dangerous tasks is ethically complex since it can endanger subjects during tests. It then becomes difficult to analyze the tasks and design solutions without considering the use of simulation tools and/or meaningful but safe mock-ups. Simulation tools are well suited to systematically evaluate and optimize design choices related to the physical and control architecture of potential robotic assistant solutions[7].

In this work, a wood shaper simulation tool is proposed (section III). This simulator relies on a physical model of the system (section II) which accounts for the wrenches applied by the human operator, the cutting tool as well as a candidate robotic solution. This simulator is used especially in accidental situations. It is also used to evaluate the pertinence of a the proposed control solutions. The proposed approach to safety is to control the robot with the reduction of the amplitude of disturbances induced by a local modification of the nature of the wood (typically a node) in mind (section IV). A physical mock-up of the system is also proposed and the performances of the robotic solution obtained for different tuning of the controller are presented and analysed (section V).

\section{TASK MODELING}

\section{A. Equilibrium of the wooden part}

The work environment during a milling task consists of a cutting tool rotating at high speed around an axis, $\boldsymbol{z}_{\boldsymbol{R}}$, normal to an horizontal work table that serves as a support for the wood part and is defined by a plane $\mathcal{P}$. A rail-shaped guide associated to an axis $\boldsymbol{x}_{\boldsymbol{R}} \in \mathcal{P}$ is attached to the work table. 


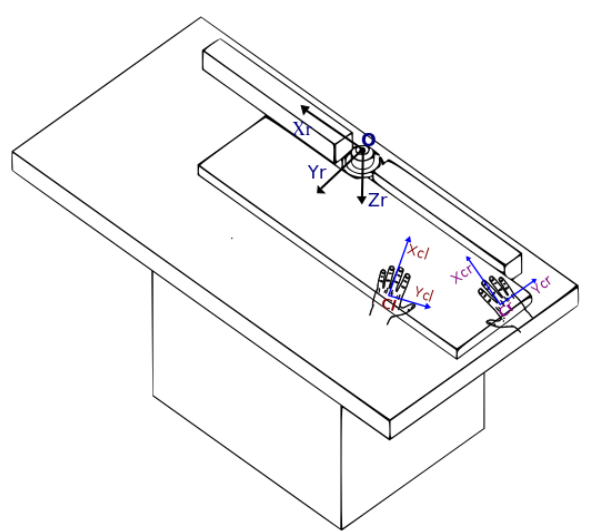

Fig. 2: Schematic representation of a wood shaper.

Let $\{\mathcal{R}\}=\left\{O, \boldsymbol{x}_{\boldsymbol{R}}, \boldsymbol{y}_{\boldsymbol{R}}, \boldsymbol{z}_{\boldsymbol{R}}\right\}$ represent a chosen orthonormal frame fixed in the workspace which origin is at the intersection of $z_{R}$ and plane $\mathcal{P}$.

The part is subject to a set of forces and moments applied by the operator and the cutting tool during the milling process. This corresponds to the more general situation of an object being subject to different external wrenches. The contact point associated to wrench $\boldsymbol{w}_{i}=\left(\begin{array}{cc}\boldsymbol{f}_{i}^{T} & \boldsymbol{m}_{i}^{T}\end{array}\right)^{T}$ (with $\boldsymbol{f}_{i} \in \mathbb{R}^{3}$ and $\boldsymbol{m}_{i} \in$ $\mathbb{R}^{3}$ the force and moment composing wrench $\boldsymbol{w}_{i}$ ) is denoted $C_{i}$ and is defined by the vector $\boldsymbol{c}_{i} \in \mathbb{R}^{3}$. A frame $\{\mathcal{C}\}_{i}$, with axes $\left\{\boldsymbol{x}_{\boldsymbol{i}}, \boldsymbol{y}_{\boldsymbol{i}}, \boldsymbol{z}_{\boldsymbol{i}}\right\}$ is also defined at this point. In the case of the wrenches applied by the operator, $\boldsymbol{x}_{\boldsymbol{i}}$ is colinear to the human forearm axes and directed toward the object and $\boldsymbol{y}_{\boldsymbol{i}}$ is parallel to the support table plane. The nature of the wrench applied by the tool is introduced in section II-B.

In order to derive the equilibrium conditions for the part, let express all wrenches at point $O$ and in frame $\{\mathcal{R}\}$

$$
\boldsymbol{w}_{i, O / \mathcal{R}}=\underbrace{\left(\begin{array}{cc}
\boldsymbol{R}_{i} & \mathbf{0} \\
\boldsymbol{R}_{i} \boldsymbol{S}\left(\boldsymbol{C}_{i}\right) & \boldsymbol{R}_{i}
\end{array}\right)}_{\boldsymbol{G}_{i}} \boldsymbol{w}_{i}
$$

with $\boldsymbol{S}\left(\boldsymbol{C}_{i}\right)$ is the skew-symmetric matrix corresponding to the cross-product with vector $\overrightarrow{O C}_{i}$ expressed in frame $\{\mathcal{C}\}_{i}$ and $\boldsymbol{R}_{i}$ the rotation matrix from frame $\{\mathcal{C}\}_{i}$ to frame $\{\mathcal{R}\}$.

$\boldsymbol{G}_{i}$ is the partial grasp matrix [8] which maps the contact wrench applied to the object at $\{\boldsymbol{C}\}_{i}$ to the equivalent wrench expressed at $O$ in frame $\{\mathcal{R}\}$. The complete grasp matrix $\boldsymbol{G} \in \mathbb{R}^{6 \times 6 \times n_{c}}$ of the system (where $n_{c}$ is the number of contacts) relate all external wrenches expressed at their respective contact points to the equivalent wrench expressed at $O$ in frame $\{\mathcal{R}\}$

$$
\boldsymbol{w}_{O / \mathcal{R}}=\underbrace{\left(\begin{array}{lll}
\boldsymbol{G}_{1} & \cdots & \boldsymbol{G}_{n_{c}}
\end{array}\right)}_{\boldsymbol{G}} \underbrace{\left(\begin{array}{lll}
\boldsymbol{w}_{1}^{T} & \cdots & \boldsymbol{w}_{n_{c}}^{T}
\end{array}\right)^{T}}_{\boldsymbol{w}} .
$$

The machining process results in a relative motion of the part with respect to frame $\{\mathcal{R}\}$. In order to compute the motion resulting from the application of all external wrenches, one can apply the fundamental principle of dynamics to the system, using the grasp matrix defined in eq. (2)

$$
\boldsymbol{G w}=\boldsymbol{M \gamma}+\boldsymbol{B v} .
$$

where $\gamma$ and $\boldsymbol{v}$ are respectively the acceleration and twist of the part expressed at $O$ in $\{\mathcal{R}\} . M \in \mathbb{R}^{6 \times 6}$ and $\boldsymbol{B} \in \mathbb{R}^{6 \times 6}$ are respectively the symmetric and positive mass matrix and diagonal viscous friction matrix.

Fore the sake of clarity and simplicity, and without loss of generality, it is assumed that the mass of the part is constant over the duration of the simulation, i.e the loss of mass due to the tooling process is neglected. It is also assumed that the friction between the table and the part is purely viscous. In practice dry friction is present but adding it to the model would not bring much added value to the overall proposed contribution. Finally, the weight of the part is fully compensated for by reaction forces of the table and any force applied to the part in the normal direction of the guiding rail is compensated for by reaction forces of the rail.

As a consequence, in the unassisted case, the only external wrenches considered in the model are the ones of the operator's left and right hand $\boldsymbol{w}_{l}$ and $\boldsymbol{w}_{r}$ and of the cutting tool $\boldsymbol{w}_{c}$.

\section{B. Wood cutting model}

Several wood cutting models have been developed since the 1950s. One of the first widely used models is proposed by E. Kivimaa works [9]. However, this simplified model can lead to large differences (up to 40\%) between the actual cutting forces and the modeled [10]. F Eyma et al. [11] have improved this model for 13 tropical wood species, but do not take into account the wood grain orientation. This characteristics largely impacts the magnitude of the cutting forces and, more importantly, a sudden modification of this orientation (typically with a node) represents a factor of potential accident. L. Cristovao et al. [12] account for this important effect in the wood cutting model. However their model only considers unidirectional cutting forces.

The model used in this paper takes its origin in an experimentally validated model developed for medium and low density woods [10]. This model expresses the cutting force at the edge of the cutting tool and decomposes it in two components $f_{\text {tan }}$ and $f_{n o r}$ which are respectively the cutting force tangential to the part feed velocity/trajectory (along the rail and $\boldsymbol{x}_{R}$ ) and normal to it (along $\boldsymbol{y}_{\boldsymbol{R}}$ ). This yields the relation between the cutting wrench expressed at the edge of the cutting tool, the partial grasping matrix associated to the cutting tool and the cutting wrench expressed at $O$ in frame $\{\mathcal{R}\}$ (index $\square_{c}$ stands for cutting):

$$
\boldsymbol{w}_{c, O / \mathcal{R}}=\boldsymbol{G}_{c}\left(\begin{array}{lll}
f_{\text {tan }} & f_{\text {nor }} & \mathbf{0}_{1 \times 4}
\end{array}\right)^{T} .
$$

This model depends on several parameters including the cutting velocity and the density and orientation of the wood grain. These two last parameters are essential to simulate the appearance of a node. The expression of $f_{\text {tan }}$ and $f_{\text {nor }}$ taken from [10] are recalled here

$$
\begin{aligned}
& f_{\text {tan }}=\left(a_{1}+a_{2}\left|\cos \left(\phi_{v}+a_{3}\right)\right|^{a_{4}}\right) A_{1}+\left(a_{5}+a_{6}\left|\sin \left(\phi_{v}+a_{7}\right)\right|^{a_{8}}\right) A_{2}+a_{27} \\
& f_{\text {nor }}=\left(b_{1}+b_{2}\left|\cos \left(\phi_{v}+a_{3}\right)\right|^{b_{4}}\right) B_{1}+\left(b_{5}+b_{6}\left|\sin \left(\phi_{v}+b_{7}\right)\right|^{b_{8}}\right) B_{2}+b_{27} \\
& A_{1}=a_{p}^{a_{9}} \lambda_{F}^{a_{1} 0} \rho^{a_{11}} v_{c}^{a_{12}} D^{a_{13}}\left(a_{14}-e^{m c . a_{15}}\right)^{-1} \cdot\left(a_{16}-e^{T . a_{17}}\right)^{-1} \\
& A_{2}=a_{p}^{a_{18}} \lambda_{F}^{a_{19}} \rho^{a_{20}} v_{c}^{a_{21}} D^{a_{22}}\left(a_{23}-e^{m c . a_{24}}\right)^{-1}\left(a_{25}-e^{T \cdot a_{26}}\right)^{-1} \\
& B_{1}=a_{p}^{b_{9}} \lambda_{F}^{b_{10}} \rho^{b_{11}} v_{c}^{b_{12}} D^{b_{13}}\left(b_{14}-e^{m c . b_{15}}\right)^{-1}\left(b_{16}-e^{T . b_{17}}\right)^{-1} \\
& B_{2}=a_{p}^{b_{18}} \lambda_{F}^{b_{19}} \rho^{b_{20}} v_{c}^{b_{21}} D^{b_{22}}\left(b_{23}-e^{m c . b_{24}}\right)^{-1}\left(b_{25}-e^{T . b_{26}}\right)^{-1},
\end{aligned}
$$

where $\phi_{v}$ : angle between the cutting velocity vector; $v_{c}$ and wood grains $(\mathrm{rad}) ; a_{p}$ : thickness of the cutting layer also known as uncut chip thickness $(\mathrm{mm}) ; \lambda_{F}$ : rake angle $(\mathrm{rad}) ; \rho$ : cutting edge dullness $(\mu m) ; v_{c}$ : cutting velocity $(\mathrm{m} / \mathrm{s}) ; D$ : wood density $\left(\mathrm{kg} / \mathrm{m}^{3}\right) ; \mathrm{mc}$ : moisture content $(\%) ; T:$ temperature of the wood $(K) ; a_{j}, b_{k}$ : coefficients determined by experimental identification in [10]. 


\section{Modeling of the human operator}

Modeling the behaviour of human operator realizing an artisanal task such as wood milling is complex. In this work, several assumptions are made in order to obtain a model which level of complexity is appropriate given the exploratory nature of our study. While it does not seem desirable to include a complex musculoskeletal and motor control model of the human in this first study, the considered model should still account for the specificities of the task to be achieved as well as for some of the limits of the human in terms of applicable wrenches.

Regarding the task specificities, expert teachers from a training centre for apprentice carpenters in Bordeaux, France (namely the BTP-CFA Gironde) have provided some general indications regarding the use a wood shaper. First, the motion of the part during the cutting phase must be continuous, with constant velocity, in order to ensure a good cutting quality. Second the right hand (assumed to be the hand close to the guiding rail) is placed at the end of the part and provides wrenches mostly dedicated to its motion (along $\boldsymbol{x}_{\boldsymbol{R}}$ ) while the left hand is placed on the side of the part and provides wrenches mostly dedicated to the lateral stabilization of the part (along $\boldsymbol{y}_{\boldsymbol{R}}$ )

Regarding human capabilities in terms of producible wrench at the hands, the work in [13] provides information regarding the maximum force a male adult can produce with his hand in a pushing motion both in the direction of his forearm as well as in the direction tangential direction. The work in [14] also provides maximum values for the rate of variation of these forces induced by human motor reflexes and the dynamic of muscle contraction.

Given these elements, the wrenches produced by the human operator during a wood milling task are computed in order to servo the velocity of the part in the feed direction $\boldsymbol{x}_{\boldsymbol{R}}$ given the other external wrenches applied to the part. Assuming that an experienced human operator trains a mental model of the part being milled and can estimate, through proprioception and contact with the part, all external wrenches at work, the ideal wrenches to produce using both hands can be computed using eq. (3) as:

$$
\boldsymbol{G}_{w_{r}} \boldsymbol{w}_{r}+\boldsymbol{G}_{w_{l}} \boldsymbol{w}_{l}=\boldsymbol{M} \boldsymbol{\gamma}^{*}+\boldsymbol{B} \boldsymbol{v}-\boldsymbol{G}_{w_{c}} \boldsymbol{w}_{c}
$$

where:

- $\boldsymbol{G}_{w_{i}}$ and $\boldsymbol{w}_{i}$ are the the partial grasping matrix for the wood shaper, the right an left hands $(i=\{c, r, l\})$ respectively;

- $\boldsymbol{v}$ and $\boldsymbol{w}_{c}$ are the twist of the part and the wrench applied by the cutting tool on the part respectively;

- $\gamma^{*}$ is the control acceleration which is computed using a PI controller between the twist of the part $\boldsymbol{v}$ and the desired twist $\boldsymbol{v}^{*}$. The components of $\boldsymbol{v}^{*}$ are 0 except in the $\boldsymbol{x}_{\boldsymbol{R}}$ linear direction which is the feed direction for the part.

The bounds on the hand wrenches are written

$$
\begin{aligned}
& \boldsymbol{w}_{i, \min } \leq \boldsymbol{w}_{i} \leq \boldsymbol{w}_{i, \max }, \\
& \dot{\boldsymbol{w}}_{i, \min } \leq \dot{\boldsymbol{w}}_{i} \leq \dot{\boldsymbol{w}}_{i, \max }
\end{aligned}
$$

where $\boldsymbol{w}_{i, \min }, \boldsymbol{w}_{i, \max }, \dot{\boldsymbol{w}}_{i, \min }$ and $\dot{\boldsymbol{w}}_{i, \max }$ are taken from [13] and [14]. It is worth noticing that the hand pushing forces are unilateral (the operator can hardly pull the part) and the corresponding limit value $\boldsymbol{f}_{\{l, r\}, \min }$ are set to 0 .

\section{Simulation}

\section{A. Simulation principles}

The aim of the simulator is to compute the evolution of the wrenches applied by the human operator on the part given a desired part velocity $\boldsymbol{v}^{*}$, the length of wood to be milled $d$ for the part and a wood type. The initial state of the system is characterized by the initial positions of the hands in the reference frame $\{\mathcal{R}\}$ and by the initial twist of the part $\boldsymbol{v}(0)=\mathbf{0}$.

At each time step, the following computations are performed by the simulator:

1) the partial grasp matrices (see eq. (1)) are updated given the current location of the hand contact points $\boldsymbol{c}_{i}(i=\{r, l\})$ with respect to frame $\{\mathcal{R}\}$. Indeed these contact points remain constant in $\{\mathcal{C}\}_{i}$ but are moving with respect to $\{\mathcal{R}\}$;

2) the tool cutting force is updated given the current twist of the part $\boldsymbol{v}$;

3) the PI controller is updated given the current velocity of the part $\boldsymbol{v}$

4) the optimal hand wrenches are computed according to eqs. (5) to (7) (see hereafter);

5) the part acceleration $\gamma$ is computed through eq. (3)) given the computed hand wrenches $\boldsymbol{w}_{i}^{o p t}(i=\{r, l\})$, and tool cutting wrench $\boldsymbol{w}_{c}$;

6) the twist $\boldsymbol{v}$ of the part and location of the hands ${ }^{1} \boldsymbol{c}_{i}(i=$ $\{r, l\})$ with respect to frame $\{\mathcal{R}\}$ are updated through numerical integration using an Euler integration scheme for the computed acceleration of the part $\gamma$.

\section{B. Human operator wrenches optimization}

Computing the optimal hand wrenches (step 4) boils down to finding the optimal hand wrenches $\boldsymbol{w}_{r}^{o p t}$ and $\boldsymbol{w}_{l}^{\text {opt }}$ minimizing a cost function associated to eq. (5) under constraints defined by eqs. (6) and (7).

Such an optimization problem can be written as a quadratic program under linear constraints (LQP)

$$
\begin{array}{cc} 
& \left\{\boldsymbol{w}_{r}^{o p t}, \boldsymbol{w}_{l}^{o p t}\right\}=\underset{\boldsymbol{w}_{r}, \boldsymbol{w}_{l}}{\arg \min } f_{w}+f_{r e g} \\
\text { s.t. } & \text { eq. (6) and eq. (7) }
\end{array}
$$

where $f_{w}=\left\|\boldsymbol{G}_{w_{r}} \boldsymbol{w}_{r}+\boldsymbol{G}_{w_{l}} \boldsymbol{w}_{l}-\left(\boldsymbol{M} \boldsymbol{\gamma}^{*}+\boldsymbol{B} \boldsymbol{v}-\boldsymbol{G}_{w_{c}} \boldsymbol{w}_{c}\right)\right\|_{2}^{2}$ is the main cost function

and $f_{r e g}=\alpha\left\|\left(\begin{array}{ll}\boldsymbol{w}_{r}^{T} & \boldsymbol{w}_{l}^{T}\end{array}\right)^{T}\right\|_{\boldsymbol{W}_{\text {lim }}}^{2}$ is the regularization cost.

This part of the cost function is required for the optimization problem to have a unique solution. $\alpha$ is chosen $\ll 1$. In practice, this leads to a solution both minimizing $f_{w}$ as well as the weighted norm of the solution. The weighting matrix $\boldsymbol{W}_{\text {lim }}$ is chosen to account for the bounds on the achievable human wrenches eq. (6) and favour directions where the force capabilities are the largest. Such a weighting matrix can be defined as a $12 \times 12$ diagonal matrix which elements are in $\mathbb{R}^{+}$:

$$
\operatorname{diag}\left(\frac{1}{f_{r, \text { max }}^{x}} \cdots \frac{1}{m_{r, \max }^{z}}, \frac{1}{f_{l, \text { max }}^{x}} \cdots \frac{1}{m_{l, \text { max }}^{z}}\right) .
$$

This simulator is implemented in Matlab and the LQP optimization problem is solved using the quadprog solver. The simulator handle 3D models but in the specific case of this planar application a $2 \mathrm{D}$ projection is performed. Fig. 3 provides a view of the visualization associated to the simulator. All parameters introduced in the paper can be tuned. However typical simulations are performed with the values provided in table I.

\footnotetext{
${ }^{1}$ which is equivalent to updating the pose of the part.
} 


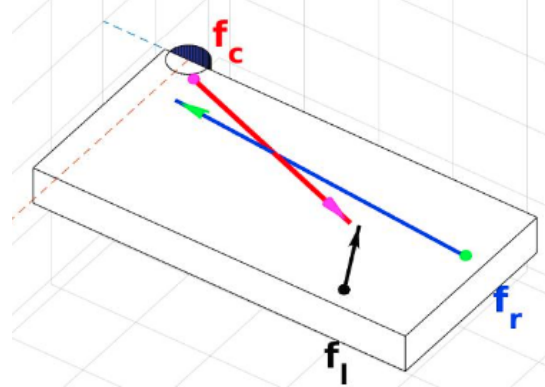

Fig. 3: Visualization of the force equilibrium between the hands and cutting forces for a desired velocity along $\boldsymbol{x}_{\mathcal{R}}$ of $0.05 \mathrm{~m} / \mathrm{s}$.

TABLE I: Typical values of the simulation parameters (after 2D projection)

\begin{tabular}{|c|c|}
\hline Simulator parameters & \\
\hline Sampling time for integration & $0.010 \mathrm{~s}$ \\
\hline Maximum simulation duration & $6 s$ \\
\hline Maximum part travel distance & $0.5 m$ \\
\hline Desired feed rate/part velocity & $0.05 \mathrm{~m} / \mathrm{s}$ \\
\hline Part characteristics & \\
\hline Size (length, width, height) & $(1.0,0.5,0.1) m$ \\
\hline Mass matrix & $\operatorname{diag}\left(1.0 \mathrm{~kg}, 1.0 \mathrm{~kg}, 2.6042 \mathrm{~kg} \cdot \mathrm{m}^{2}\right)$ \\
\hline Viscous friction with the table & $\operatorname{diag}(30.0,30.0,0.0)$ N.s. $m^{-1}$ \\
\hline Wood type & Pinus Sylvestris [9] \\
\hline Human operator characteristics & \\
\hline Initial right hand contact point & $(-0.95,0.20,0.0)_{\mathcal{R}}$ \\
\hline Initial left hand contact point & $(-0.80,0.45,0.0)_{\mathcal{R}} \quad m$ \\
\hline Tool cutting edge coordinates & $(-0.05,0.05,0.0)_{\mathcal{R}} \quad m$ \\
\hline Proportional gains & $(10.0,10.0,10.0) \quad s^{-1}$ \\
\hline Integral gains & $(10.0,10.0,10.0) \quad s^{-2}$ \\
\hline Weight of the regularization cost & $10^{-6}$ \\
\hline Maximum right hand wrench & $(160.0 \mathrm{~N}, 15.0 \mathrm{~N}, 13.0 \mathrm{~N} . \mathrm{m}) \mathcal{C}_{r}$ \\
\hline Minimum right hand wrench & $(0.0 N,-15.0 N,-13.0 N . m) \mathcal{C}_{r}$ \\
\hline Maximum left hand wrench & $(13.0 N, 133.0 N, 13.0 N . m)_{\mathcal{C}_{l}}$ \\
\hline Minimum right hand wrench & $(-13.0 \mathrm{~N}, 0.0 \mathrm{~N},-13.0 \mathrm{~N} . \mathrm{m})_{\mathcal{C}_{l}}$ \\
\hline Maximum wrench rate of change & $120 \mathrm{~ms}$ from $\boldsymbol{w}_{i, \min }$ to $\boldsymbol{w}_{i, \max }$ \\
\hline
\end{tabular}

\section{Simulating instabilities}

Two major cases of instabilities leading to accidents are generally observed by experts.

The first case of instability occurs when the cutting tool hits a node. Cutting forces suddenly increase and the carpenter's limited reflexes and strength (eqs. (6) and (7)) may not be sufficient to instantaneously compensate for the unexpected increase in force. This leads to a transient inversion of the direction of the velocity of the part and an associated backward motion. The part hits the hands of the carpenter, potentially his arms and body.

The second case of instability occurs when the cutting tool is in contact with a wood node and suddenly comes out of it. Cutting forces suddenly decrease and, due to similar limitations, the carpenter may not be able to instantaneously compensate for the unexpected increase in force. This leads to a transient increase of the velocity of the part and a faster motion of the part, and as a consequence of the hands of the carpenter, towards the cutting tool. The consequences can be dramatic, including complete severing of several fingers and/or deep cuts of the hands. Besides the more severe consequences on the human operator, this instability is harder to compensate for because of the unilateral nature of the constraint on the force for the right hand in the $\boldsymbol{x}_{\mathcal{R}}$ direction and of the very low force exertion capability of the left hand in the $x_{l}$ direction.

Using this simulator, these two typical instabilities can be simulated by introducing nodes in the wood, i.e by varying the angle of the grain of the wood with respect to the feeding direction. Simulation results corresponding to such instabilities are presented in fig. 4 (a) and (b) for the first case of instability (cutting tool entering a node) and fig. 4 (c) and (d) for the second one (cutting tool leaving a node).

On fig. 4 (a) and (b), slightly before $t=3.1 \mathrm{~s}$, the simulated apparition of a node leads, as expected, to an instantaneous ${ }^{2}$ increase of the cutting force from $f_{t a n}=-39.8 \mathrm{~N}$ to $f_{\tan }=$ $-132.2 N$. This induces an increase of the required force $f_{r}^{x}$ for the right hand in the direction of motion of the part (along $\boldsymbol{x}_{\mathcal{R}}$ ). The limit on the rate of evolution of the wrench applicable by the hands is such that the part experiences a fast backward motion ( $25 \mathrm{~cm}$ and peak at $v^{x}<-1.0 \mathrm{~m} / \mathrm{s}$ ) before getting back on track once the hands have reached the required level of force. Similarly, on fig. 4 (c) and (d), the simulated disappearance of a node leads to an instantaneous decrease of the cutting force from $f_{\text {tan }}=-112.0 \mathrm{~N}$ to $f_{\text {tan }}=-20 \mathrm{~N}$. Analogously to the previous case, this generates a fast forward motion (same amplitude) of the part before the motion of the part is performed at constant velocity again.

These two examples confirm the ability of the simulator to reproduce physically realist simulations of both nominal and accidental situations.

\section{RoBOTIC ASSISTANCE FOR SAFETY}

The robotic assistance provided to the user should aim at decreasing the risk of sudden modification of the velocity of the part while allowing to maintain direct physical interaction of the carpenter with the wood as well as his sensations. Candidate control modes should also imply the least possible need for external sensing devices which may not be robust enough in a dusty environment and largely increase the complexity of the overall solution.

To meet this specifications, it is proposed to use the robotic system in order to damp the transient velocity change when an instability occurs. The robot end-effector is assumed to be maintained in contact with the wood (the study of the associated technological solutions is out of the scope of this work) at an arbitrarily chosen point $\boldsymbol{C}_{a}$ (index ${ }_{a} \square_{a}$ stands for assistance). The associated wrench $\boldsymbol{w}_{a}$ and associated partial grasping matrix $\boldsymbol{G}_{a}$ can be included seamlessly in eqs. (3) and (5) and section III-B and the simulation directly includes the contribution of the robot to the total wrench applied to the part.

Assuming the use of a torque control robot (e.g. 7DOF Panda from Franka Emika), the emulation of a damping behaviour can be obtained by choosing the control torques $\tau^{c}$ as

$$
\boldsymbol{\tau}^{c}=\boldsymbol{g}(\boldsymbol{q})-\boldsymbol{J}(\boldsymbol{q})^{T} \boldsymbol{B}_{a} \boldsymbol{t}
$$

where, for the chosen robot: $t$ is the twist of the end-effector, directly related to $\boldsymbol{v}$, the twist of the part; $\boldsymbol{q}$ is the generalized coordinates vector $; \boldsymbol{g}(\boldsymbol{q})$ is the gravity compensation torque ; $\boldsymbol{J}(\boldsymbol{q})$ is the end-effector Jacobian matrix and $\boldsymbol{B}_{a}$ is a damping matrix. This matrix is typically diagonal with components defined in $\mathbb{R}^{+}$). The damping coefficients can for example be chosen to be higher in Cartesian directions requiring more damping. In this work they are chosen, without loss of generality, to be equal $\left(b_{a}\right)$. In the

\footnotetext{
${ }^{2}$ in practice this increase is not instantaneous but can be considered as such given the reaction time of the human.
} 


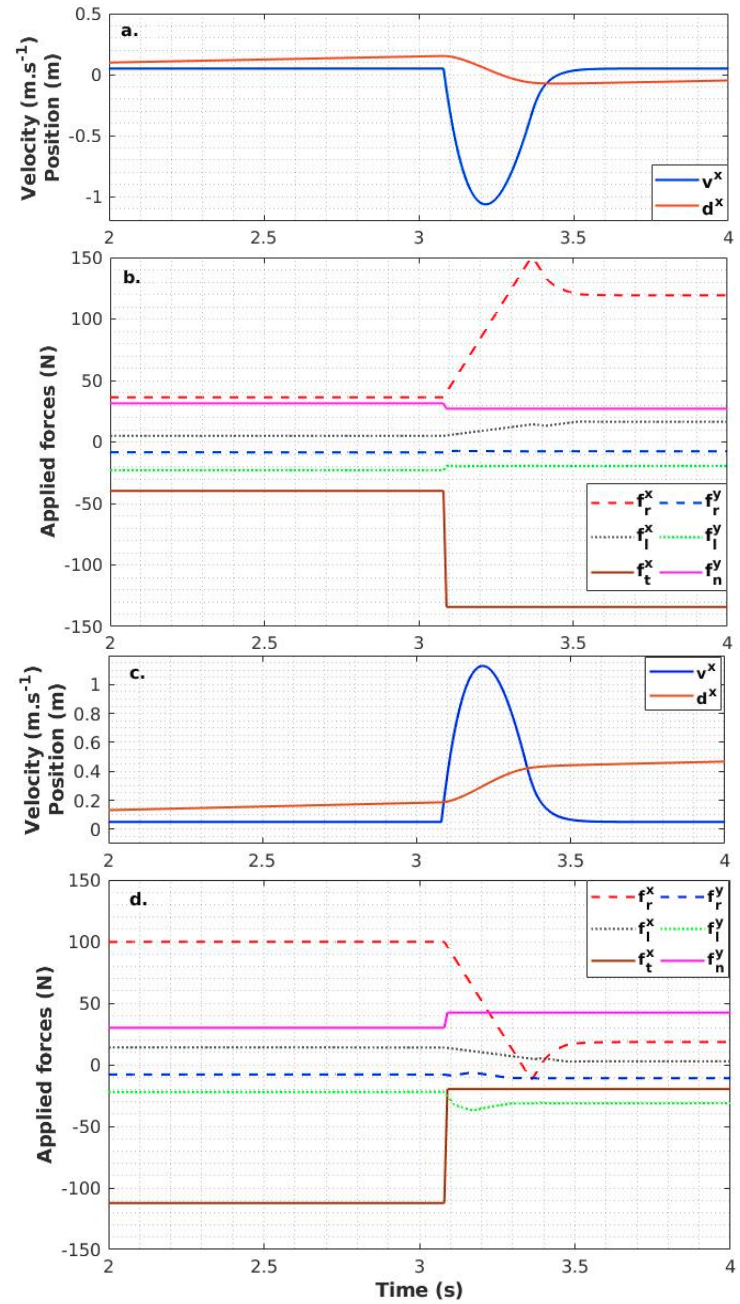

Fig. 4: Results of the simulation of an instability due to the cutting tool entering ((a) and (b)) and leaving ((c) and (d)) a node. Figures (a) and (c) plot the evolution with respect to time of the distance travelled by the part along $\boldsymbol{x}_{\mathcal{R}}$ as well as the corresponding part velocity in the same direction. Figures (b) and (d) provide the evolution of the right and left hands forces as well as of the cutting forces in the XY plane of the reference frame.

context of this work these directions are obviously $\boldsymbol{x}_{\mathcal{R}}$ and $\boldsymbol{y}_{\mathcal{R}}$. With this controller, the robot wrench applied to the part, is given in the robot end-effector frame by

$$
\boldsymbol{w}_{a}=-\boldsymbol{B}_{a} \boldsymbol{t} .
$$

\section{EXPERIMENTATION AND RESULTS}

To confirm the observations made in simulation on the impact of a sudden variation of the cutting force on the part motion, an experiment has been designed to reproduce one of the two cases of instability described in section III-C. It was considered more interesting to focus on the case where the cutting tool exits a node given the higher risk of induced dramatic accident (potential contact of the hands with the cutting tool).

While conducting experiments using a real wood shaper would have been possible, it was judged safer to realize this first experi- ment using a simplified mock-up that cannot cause any harm to the user (see fig. 5).

\section{A. Mock up description}

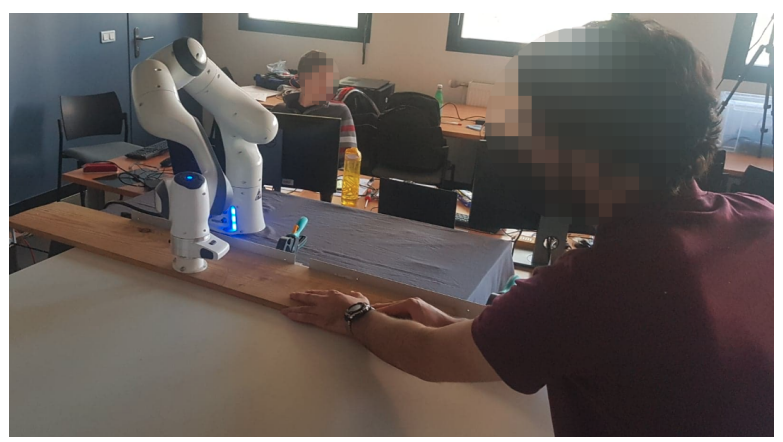

Fig. 5: Experimental mock-up including a table, a guiding rail, a wooden board, the Panda robot and one of the three subjects. The pulley system and load used to produce the emulated cutting force are placed under the table.

To remove any source of risk, the cutting force is emulated using a constant horizontal force of $75 \mathrm{~N}$ applied to a wooden board. This force is produced using a load attached via a pulley to the board. So, for the wooden board to move forward, the user must apply a pushing force of at least $75 \mathrm{~N}$. The motion of the board is guided by a rail similar to the ones used on real wood shapers. The sudden force drop due to the cutting tool exiting the node is emulated by an instantaneous reduction of the dragging force to $25 \mathrm{~N}$. Note that to guarantee the safety of the test subjects, the force implied in the process are reduced compared to the more realistic cases simulated and presented in section III-C.

To verify the hypothesised benefits of a robotic assistance, a 7-DOF torque controlled robot (Panda from Franka Emika) is attached to the wooden part. The control law described by eq. (10) is used at all time with damping values in the XY plane of the table ranging from 0 to $600 \mathrm{~N} . \mathrm{s} . \mathrm{m}^{-1}$. The robot being rigidly attached to the wooden board, it provides access to its position and velocity.

\section{B. Protocol}

Three healthy male subjects (none of them is co-author of this paper) are asked to move the wooden board part along the rail at a constant velocity. Indications on where to position their hands are given to them. The subjects first perform a few trials without accident emulation to get accustomed to the dynamics of the wooden board subjected to the emulated cutting force. After this short training, each subject realizes five recorded experiments with accident emulation, each time with a different value of the damping coefficient. For subject 3 , an additional downwards constant vertical force of $50 \mathrm{~N}$ is applied by the robot to the wood when the rate of change of the planar velocity is greater than $20 \mathrm{~m} / \mathrm{s}^{2}$.

\section{Results and discussions}

Figure 6 provides several information regarding the performed experiments. Figure 6 (a) (blue curve) compares the velocity profile of the part in the $\boldsymbol{x}_{\mathcal{R}}$ direction when the disturbance occurs for subject 1 for different damping values. The effect of damping on the reduction of the peak velocity is clearly positive in terms of reduction of the forward motion of the hands induced by the wood disturbance. Interestingly, considering a similar type of instability 

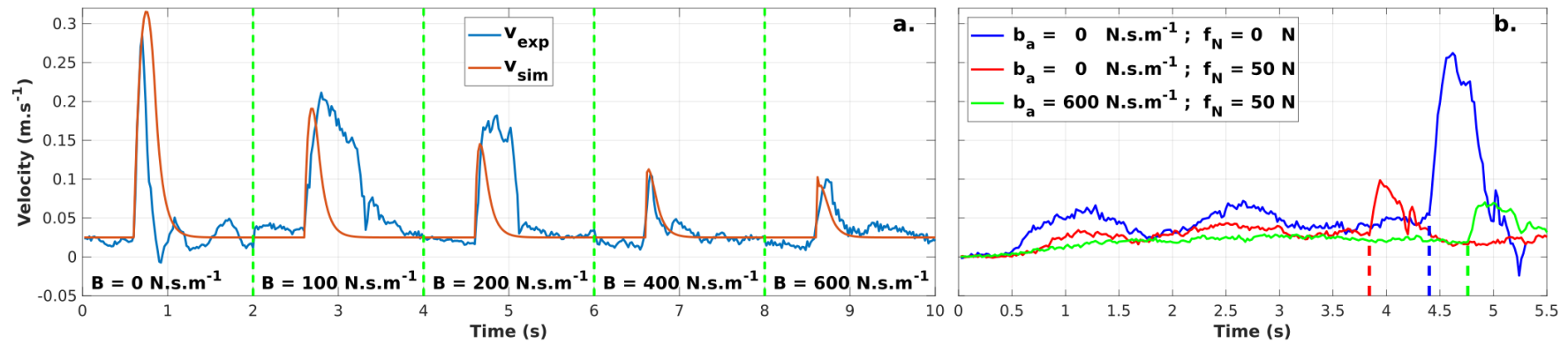

Fig. 6: (a) shows the velocity evolution of the wooden part for different damping values provided by the robot (from the unassisted case $b_{a}=0 \mathrm{~N} \cdot \mathrm{s} \cdot \mathrm{m}^{-1}$ to the maximum damping applied value $b_{a}=600 \mathrm{~N} \cdot \mathrm{s} \cdot \mathrm{m}^{-1}$ ). The blue curve represents the data measured during the experiment of the first subject and the red curve the one generated by the simulator. (b) describes the evolution of the velocity of the wooden part for subject 3, i.e. in the case where a $50 \mathrm{~N}$ vertical force is also triggered in case of disturbance (red and green curves). The blue curve corresponds to the fully unassisted case.

in simulation leads to a similar effect (red curve), both qualitatively and quantitatively. As expected, the larger the damping value, the more efficient the reduction of the peak velocity is. Nevertheless, one should note that, quite logically, increasing the damping has a slightly noticeable effect on the effort required by the simulated human to maintain the part velocity at the desired value: the steady-state force to be applied by the right hand along $\boldsymbol{x}_{R}$ increases from 72 to $86 N$ when the damping increases from 0 to $600 N . s . m^{-1}$.

Finally, fig. 6 (b) presents some of the results for subject 3 for which the control action of the robot is both a damper in the planar direction and a brake in the vertical direction. This constant braking action which is tuned to be triggered in case of abrupt acceleration induce in practice an increase of the friction between the wooden board and the table and thus reinforces the primary damping effect. Three cases are exposed: the completely unassisted one (blue curve), the one where only the vertical breaking force is applied (red curve) and the one where maximum damping is applied in the planar direction additionally to the vertical braking action. While the case of maximum planar damping is rather comparable to the results obtained with subject 1 , the pure vertical braking action (red curve) produces, quite interestingly, a very efficient reduction of the effect of the wood disturbance.

The overall obtained results are summarized in table II. This results are in-accordance with the conclusions drawn for fig. 6 and also confirm that the addition of a vertical force significantly reduces disturbance peaks. This is of interest as this vertical braking action 1) may not require the robot to be attached to the part (this is clearly an advantage in terms of applicability) and 2) is only triggered in case of disturbance and does not induce a permanent increase of the required pushing force of the operator. It may however be more complex to tune than the damping action. Further comparisons of these two strategies require a more longitudinal study which could be the topic of some future work.

\section{CONCLUSION}

This article presents a robotic assistance solution that secures a wood milling task, well known as dangerous. A simulated physical model shows the interaction wrenches between the human, the tool and the wooden part as well as their dynamics during the task. This model allows to analyse accidentogenic cases and is also used to design a proper control strategy for the assistive robot. The proposed approach is validated experimentally by testing, on
TABLE II: Effect of the damping coefficient on the velocity peak for a desired velocity of the part $\boldsymbol{v}^{*} \cdot \boldsymbol{x}_{R}=0.025 \mathrm{~m} / \mathrm{s}$. The velocity peak is expressed in $\mathrm{m} / \mathrm{s}$. The relative reduction $(\%)$ of the peak height with respect to the the unassisted case $\left(b_{a}=0 N . s . m^{-1}\right)$ is also provided. The results are presented both for the simulation and for the three subjects who performed the experiment. An additional $50 \mathrm{~N}$ vertical braking action is triggered for Subject 3 when the acceleration of the part is greater than $20 \mathrm{~m} / \mathrm{s}^{2}$. For this subject, the value of the velocity peak in the case with no damping is provided for the fully unassisted case, i.e. the vertical force is not triggered. The value of the velocity peak corresponding to zero damping and the triggered vertical force is $0.15 \mathrm{~m} / \mathrm{s}$ which yields a reduction of $42.3 \%$ of the peak.

\begin{tabular}{c|c|cc|cc|cc|cc}
\hline$b_{a}$ & 0 & 100 & $\%$ & 200 & $\%$ & 400 & $\%$ & 600 & $\%$ \\
\hline \hline Sim. & 0.33 & 0.19 & $42.4 \%$ & 0.14 & $57.6 \%$ & 0.11 & $66.7 \%$ & 0.10 & $69.7 \%$ \\
\hline S1 & 0.29 & 0.21 & $27.6 \%$ & 0.18 & $37.9 \%$ & 0.10 & $65.5 \%$ & 0.10 & $65.5 \%$ \\
\hline S2 & 0.26 & 0.18 & $30.8 \%$ & 0.15 & $42.3 \%$ & 0.14 & $46.2 \%$ & 0.12 & $53.8 \%$ \\
\hline S3 & 0.26 & 0.10 & $61.5 \%$ & 0.10 & $61.5 \%$ & 0.09 & $65.4 \%$ & 0.07 & $73.1 \%$ \\
\hline
\end{tabular}

a 7-DOF robot, a Cartesian space damping controller to secure an emulated accident on an harmless mock-up for three healthy subjects. The experimentation confirms the results obtained in simulation and the pertinence of both the simulator and the control approach.

These promising results suggest that simulation can be used to design advanced control architectures including virtual walls around the cutting-tool as well as adaptive damping that would account for the operator know-how and level of expertise. Improvement of the simulated human model could also permit the design of the assistance solution with ergonomics in mind.

Overall, this work brings to light the artisanal field that can clearly gain a lot in terms of safety and worker's health preservation through the use collaborative robots.

\section{ACKNOWLEDGEMENT}

This research work is funded by Inria and the French region Nouvelle-Aquitaine under convention $\mathrm{N}^{\mathrm{o}}$ 2018-1R50130. The authors also thank the professional training centre "BTP-CFA Gironde" which provided human and material resources for the development of this work. 


\section{REFERENCES}

[1] J. Hester, Lipscomb, J. Nolan, D. Patterson, V. Sticca, and D. J. Myers, "Safety, incentives, and the reporting of work-related injuries among union carpenters," American Journal of Industrial Medicine, vol. 57, pp. 88-102, 2013.

[2] IRIS-ST, "Fiche sinistralité - Menuiserie," France, 2016. [Online]. Available: http://www.iris-st.org/upload/Fiche\%20AT\%20MP\%20$\% 20$ menuiserie\%202016\%20charte.pdf

[3] A. Ajoudani, A. M. Zanchettin, S. Ivaldi, A. Albu-Schäffer, K. Kosuge, and O. Khatib, "Progress and prospects of the human-robot collaboration," Autonomous Robots, vol. 42, no. 5, pp. 957-975, 2018.

[4] H.-D. Lee, B.-K. Lee, W.-S. Kim, J.-S. Han, K.-S. Shin, and C.S. Han, "Human-robot cooperation control based on a dynamic model of an upper limb exoskeleton for human power amplification," Mechatronics, vol. 24, no. 2, pp. 168-176, 2014.

[5] K.-Y. Lee, S.-Y. Lee, J.-H. Choi, S.-H. Lee, and C.-S. Han, "The application of the human-robot cooperative system for construction robot manipulating and installing heavy materials," in 2006 SICEICASE International Joint Conference. IEEE, 2006, pp. 4798-4802.

[6] X. Lamy, F. Collédani, F. Geffard, Y. Measson, and G. Morel, "Overcoming human force amplification limitations in comanipulation tasks with industrial robot," in 2010 8th World Congress on Intelligent Control and Automation. IEEE, 2010, pp. 592-598.

[7] P. Maurice, V. Padois, Y. Measson, and P. Bidaud, "Human-oriented design of collaborative robots," International Journal of Industrial Ergonomics, vol. 57, pp. 88-102, 2017.

[8] D. Prattichizzo and J. C. Trinkle, "Grasping," in Springer handbook of robotics. Springer, 2016, pp. 955-988.

[9] E. Kivimaa et al., "Cutting force in woodworking." Cutting force in woodworking., no. $18,1950$.

[10] B. Porankiewicz, C. Tanaka et al., "Cutting forces by peripheral cutting of low density wood species," BioResources, vol. 2, no. 4, pp. 671681, 2007.

[11] F. Eyma, P.-J. Méausoone, and P. Martin, "Study of the properties of thirteen tropical wood species to improve the prediction of cutting forces in mode b." Annals of forest science, vol. 61, no. 1, pp. 55-64, 2004.

[12] L. Cristóvão, O. Broman, A. Grönlund, M. Ekevad, and R. Sitoe, "Main cutting force models for two species of tropical wood," Wood Material Science \& Engineering, vol. 7, no. 3, pp. 143-149, 2012.

[13] NASA, "Man-systems integration standards," 2018. [Online]. Available: https://msis.jsc.nasa.gov/Volume1.htm

[14] R. Lee and W. Tatton, "Long latency reflexes to imposed displacements of the human wrist: dependence on duration of movement," Experimental brain research, vol. 45, no. 1-2, pp. 207-216, 1982. 